\title{
Okkam - advanced morphological approach as method for computer aided innovation (CAl)
}

\author{
Dmitry Rakov ${ }^{1, *}$ \\ ${ }^{1}$ Institute of Engineering Science named after A.A. Blagonravov of the Russian Academy of \\ Sciences, Moscow, Russia
}

\begin{abstract}
This paper presents the use of Advanced Morphological Approach based for analysis, generation and synthesis of new engineering solutions as part CAI (computer-aided innovation). CAI is developing as a response to the higher demands of science and industry to the innovative level of new products. The developed approach and a software Okkam is to create a sequence of operations, comparison and selection of rational variants set by means of a series of successive procedures. The specifics of structural synthesis consist of the discreteness of variables, the presence of conditionally logical limitations and the need to work with multiple conflicting criteria. Key objective is to find a solution space with the potential to fulfil the top level engineering systems requirements. Usage of cluster analysis, set theory, set of rules allows to identify the clusters of innovative systems combining high performance potential with robustness regarding requirement changes and design uncertainties. Implementation of the approach is demonstrated by the example of the analysis and synthesis of communications devices. These studies verify the weighty potential of the pro-posed approach compared to present methods.
\end{abstract}

\section{Introduction}

The design of a device (system, process) is a set of two main tasks: the definition of (a) the structure (structural synthesis) and (b) of parameter range for the synthesized structure (parametric synthesis or parametric optimization). The solution strategies for these two tasks are different: Parametric synthesis tasks are usually reduced to the determination of solutions satisfying the metric criteria, making them formally resolved while the task of structural synthesis is absolutely different. The latter cannot be generally allocated to the class of formally solvable problems. The result of structural synthesis is the choice of the rational structure of the object. Structural synthesis requires to work with uncertain structural connections, non-metrical attributes of the structure elements and quality criteria. The objective function of a structural synthesis does not correspond to the main requirements of usual optimization methods because (1) it is discontinuous or cannot always be determined; (2) it exists in operator notation; (3) it is not based on analytical expressions; (4) it is not differentiable, not unimodal, not separable, and not additive [1].

\footnotetext{
* Corresponding author: rdl@mail.ru
} 
The solution of the structural synthesis task is the main and exclusive subject of the researcher's creative activity.

The specifics of structural synthesis tasks consist of the discreteness of variables and presence of conditionally logical limitations. To this we will add the need to work with multiple conflicting criteria. The essence of project research consists in the purposeful alteration of characteristic values for variants improving the initial ones. The very notion of "the best" in project tasks is undefined and vague, since a number of criteria are not quantifiable and/or conflict with each other. The main difficulty in the search for a designed of an ES is the uncertainty of the results due to incomplete information on evaluation criteria [2].

The structure of systems represents the most concrete way of representing a system, and the most concrete stage in generating systems during the design process [3]. In the work was examined three conceptual design levels of an Engineering Systems (ES) [4]. The characteristics of the synthesized systems on the third level of an optimization can be improved in average by $10-15 \%$ (figure 1). At level 1 and 2 the characteristics can be improved in average by $30-35 \%$ and sometimes more.

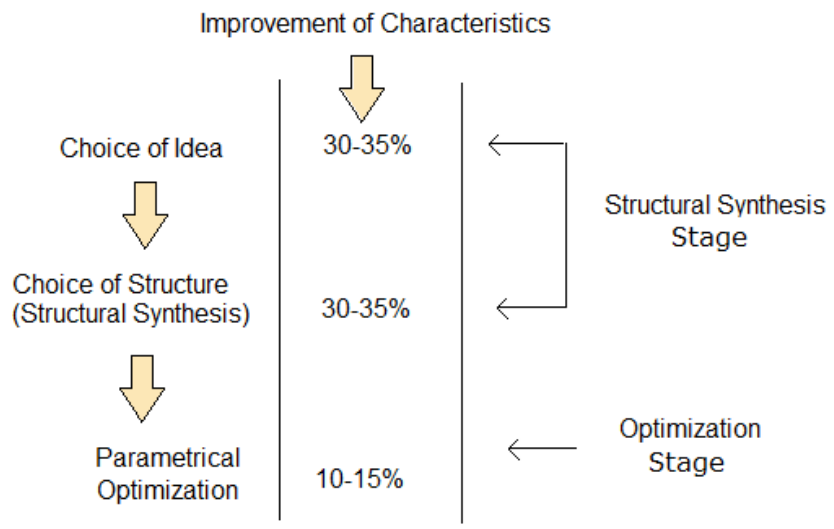

Fig. 1. Improving system performance depending on conceptual design levels

The ES structure is composed of a set of elements (constructive, functional or technological) and a set of their relationships. During the stage of structural synthesis of a new ES intuitive (Brainstorming, Mind Mapping, TRIZ, Synectics etc.) and discursive (morphological analysis, cause-and-effect diagram, Osborn-Checklists etc.) techniques can be used.

The most common method among the discursive techniques is morphological analysis [5]. By frequency of use, morphological methods are the first among ranks of discursive approaches. Thus, according to statistics compiled in 2009 by German scientists, the total number of companies using the morphology is more than $40 \%$, while regular use is done by more than $20 \%$ [6]. Morphological synthesis is regarded as a methodology to streamline the problem to be solved. Morphological analysis is a method developed by F. Zwicky for exploring all the possible solutions to a multi-dimensional, non-quantified problem complex [7]. Zwicky applied this method to such diverse tasks as the classification of astrophysical objects and the development of jet and rocket propulsion systems. More recently, morphological analysis has been extended and applied by a number of researchers in the USA and Europe in the field of future studies, engineering system analysis and strategy modeling.

Most discursive approaches to structure analysis and synthesis of ES are based on morphological models, which, in this case, have several disadvantages: the enormous 
power of the morphological set of solutions, the problems of choosing rational options and their interaction with the external environment [7]. The power of the morphological set can reach millions of possible solutions. In general, classical morphological models are inappropriate for large parameter studies, e.g. in flight systems optimisation.

At present, there are many methods to search and synthesize solutions based on the morphological analysis in in a variety of physical and engineering areas [8]. The power of the morphological set can reach millions of possible solutions (figure 2). In general, classical morphological models are inappropriate for large complex studies. Among the problems of application of classical methods of morphological analysis are most often mentioned: poor access to support software which can address the combinatorial explosion generated by multi-parameter problem spaces inherent in the use of morphological analysis; insufficiently flexible processes that address users' operational constraints; seen to be overly generic, disguising identification of specific application areas of interest [9].

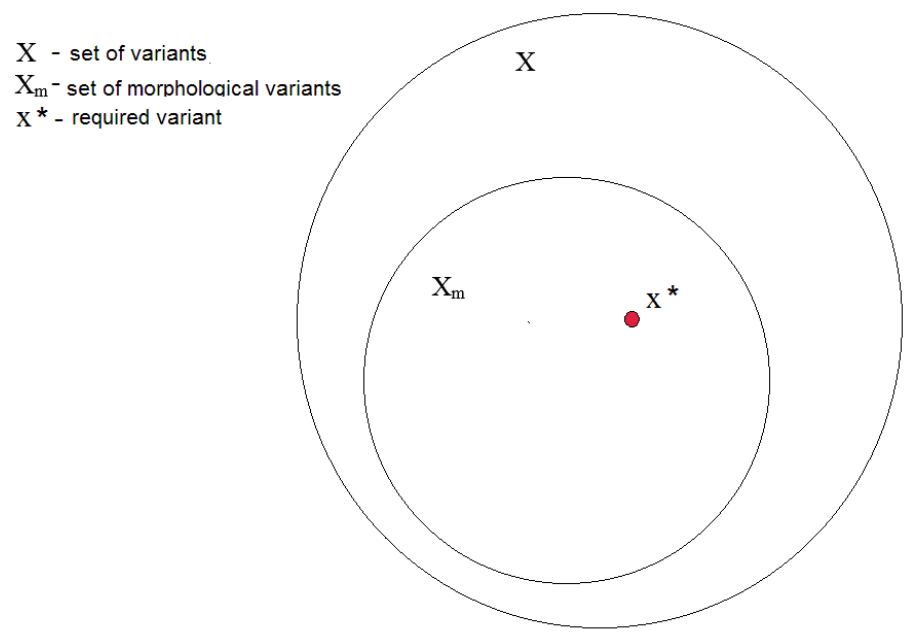

Fig. 2. Classical search for the required variant $x$ *in the morphological set Xm

One solution of this problem, in our view, consists in using models of the clustering and set theory [10]. In this respect, it seems appropriate to resort to advanced morphological approach (AMA) [11].

\section{Advanced morphological approach}

In the proposed AMA method and software Okkam, conceptual design is conducted in follow steps (1) (figures 3,4) synthesis of the morphological matrix, creating a system of criteria (2) weighting of options (3) and determination of the reference variants (4), generation (5) and selection of variants (6) clustering of variants based on similarity measure and creation the solution space (7) analysis of the clusters and solutions (8) analysis of the design risk, variants and selection (9) synthesis of anticipation models, parametric modeling and optimization stage (10). 


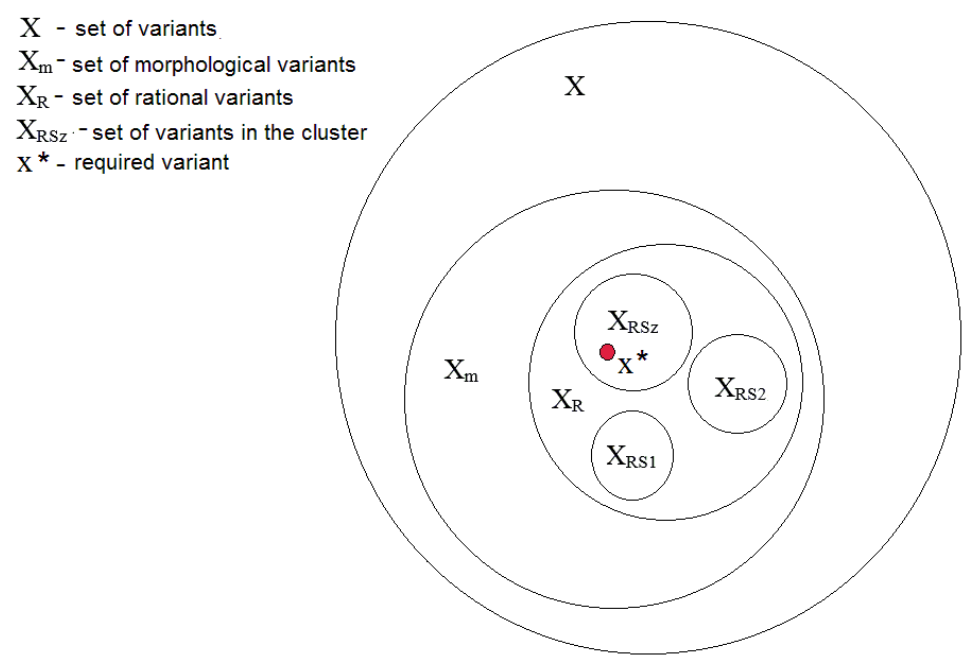

Fig. 3. AMA for the required variant $x^{*}$ search in the clusters set $X_{R S}$

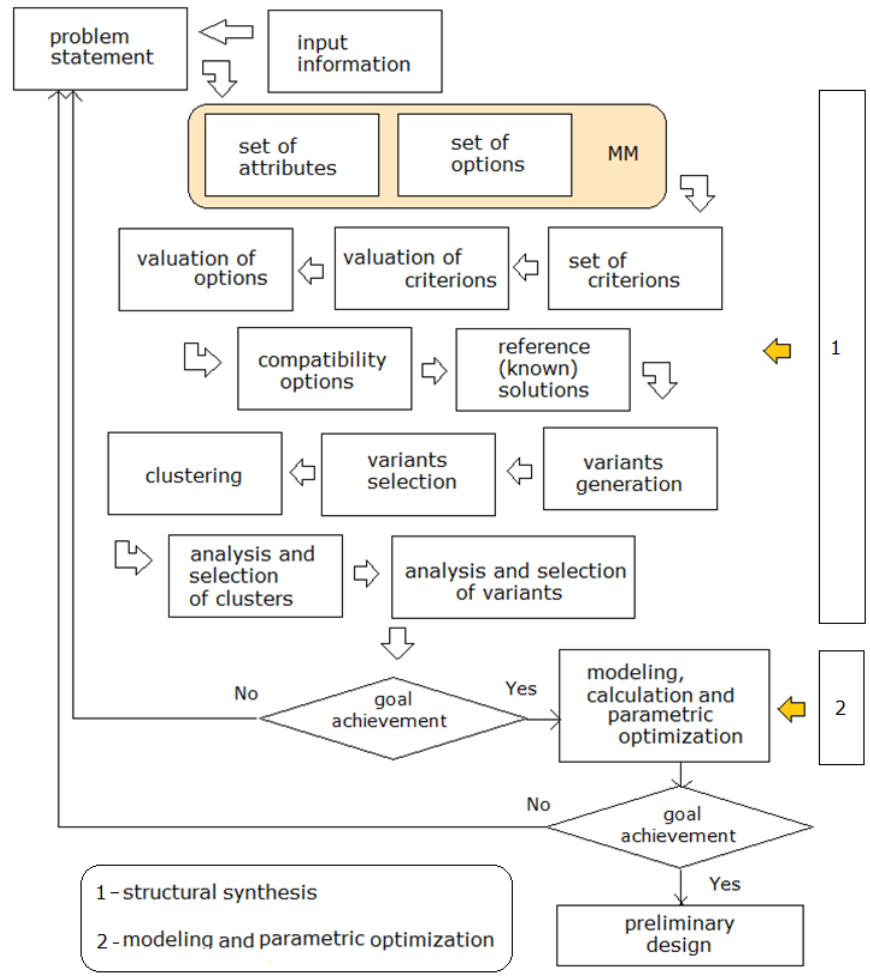

Fig. 4. AMA block diagram (program Okkam)

Let ES synthesized with the desired degree of detail is compared with the vector $\mathrm{x}=\left(\mathrm{x}_{1}\right.$, $\left.\mathrm{x}_{2}, \ldots, \mathrm{x}_{\mathrm{n}}\right)$ of dimension $\mathrm{n}$, that characterizes the set of system attributes. This $\mathrm{x} \in \mathrm{X}_{\mathrm{m}}$, where $\mathrm{X}_{\mathrm{m}}$ is morphological set of potential variants, and the synthesized variant $\mathrm{X}^{*}$ belongs to a set of $\mathrm{X}_{\mathrm{m}}$. External conditions affecting the functioning of ES, are known and fixed, and the 
criterion of operation of ES is a function $\mathrm{F}(\mathrm{x})$ and required variant $\mathrm{x} * \mathrm{CX}_{\mathrm{m}}$. The task is to find

$$
x^{*}=\arg \max _{x \in X_{M}} F(x)
$$

The synthesis process provides for the establishment of morphological matrix (MM) and supplementary information tables. The main attributes describing the performance of the object are highlighted. Depending on the task, the experts select significant attributes Pn out of a set of main attributes Pp. For each attributes are chosen the options, i.e. possible options for its execution or implementation. After decomposition and analysis of ES, morphological table (MM) is formed (figure 5) and the morphological variants set is array:

$$
X_{M}=\left\{x_{M i}, i=1, n\right\},
$$

$\mathrm{n}$ is the number of variants ES in MM (capacity of morphological set), i.e. $|\mathrm{Xm}|=\mathrm{n}$; Xmi - i-th variant of the ES.

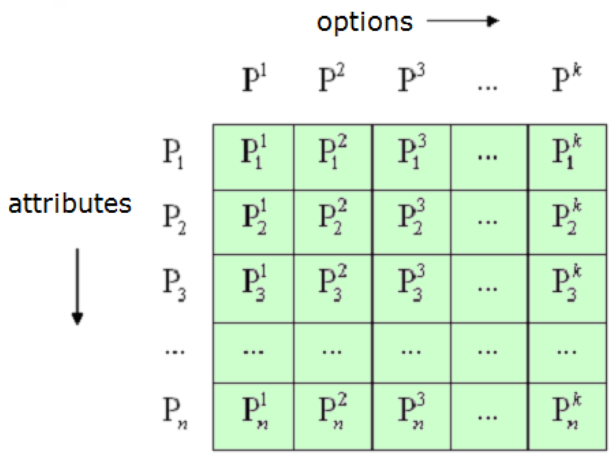

Fig. 5. Morphological Matrix

The built MM contains a multitude of reference (known solutions - from descriptions of patents, literature and technical documentation) $X_{R o}$ variants from which information tables are constructed. For example, the table "Reference Variants" is created to assess the innovations for the subsequent analysis of the degree of innovativeness of the synthesized variants. Then, a generation ES, their evaluation and initial investigations are performed. A variety of rational variants ES $X_{R v}$ is set, where $X_{R v} \leq X_{M}$. The set of rational variants is defined as the sum of them:

$$
\begin{array}{r}
X_{R}=X_{R v}+X_{R o}, \\
X_{R}=\left\{x_{R k}, k=1, p\right\},
\end{array}
$$

$p$ is a number of rational variants $X_{R}$, i.e. $\left|X_{R}\right|=p$.

Each variant $\mathrm{x}_{\mathrm{R} 1}, \mathrm{x}_{\mathrm{R} 2}, \ldots \mathrm{x}_{\mathrm{Rn}}$, contained in $\mathrm{MM}$ receives $\mathrm{U}$ evaluation depending on criterion $\mathrm{k}_{1}, \mathrm{k}_{2} \ldots \mathrm{k}_{\mathrm{m}}$, scoring options $\mathrm{z}_{11}, \mathrm{z}_{12}, \ldots, \mathrm{z}_{\mathrm{mn}}$ and criteria weight coefficients $\mathrm{w}_{1}$, $\mathrm{w}_{2}, \ldots, \mathrm{w}_{\mathrm{m}}$ (figure 6). 


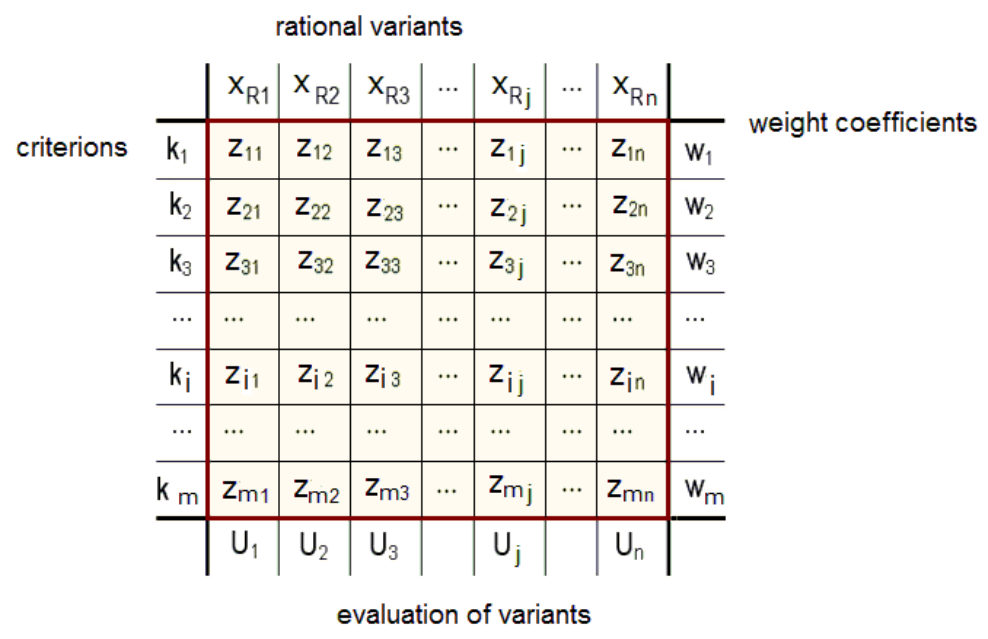

Fig. 6. Variants evaluation table

Integral assessment of each evaluation option $\mathrm{U}$ is expressed as:

$$
U=F(Z, W),
$$

$Z$ - value of estimates $Z_{i j}, i=1, m ; j=1, n$

$\mathrm{W}$ - weight value of $\mathrm{w}_{\mathrm{i}}$ criteria, $\mathrm{i}=1, \mathrm{~m}$;

$\mathrm{F}$ - function convolution of local estimates, which assigns to each of the variants the value of the assessment of $U_{n}$ is the convolution of the vector $U_{j}$. The higher the value of $U$, the more this variant is preferred, i.e. if $\mathrm{U}\left(\mathrm{x}_{\mathrm{Ri}}\right)>\mathrm{U}\left(\mathrm{x}_{\mathrm{Rj}}\right)\left(\mathrm{U}\left(\mathrm{x}_{\mathrm{Ri}}\right)\right.$ is value for variant $\mathrm{x}_{\mathrm{Ri}}$ $\left(\mathrm{x}_{\mathrm{Ri}} \in \mathrm{X}_{\mathrm{R}} \in \mathrm{X}_{\mathrm{M}}\right)$, then the variant $\mathrm{x}_{\mathrm{Ri}}$ is preferred over the variant $\mathrm{x}_{\mathrm{Rj}}$. Next clusters ES. Next clusters ES variants are formed. A $X_{R}$ set is a union of disjoint subsets (clusters) $X_{R S 1}, X_{R S 2}$, $\ldots X_{R S z}$ where $z$ is number of clusters. The set of subsets $\left\{X_{R S 1}, X_{R S 2}, \ldots X_{R S z}\right\}$ is a covering of the $X_{R}$ set, where $X_{R S i} \cap X_{R S j}=f$ and $i \neq j$. It is assumed that each variant of ES refers strictly to one cluster, i.e, there is a deterministic problem statement. Clustering is performed using the distance function with the following properties:

$$
\begin{aligned}
& \left(x_{\varepsilon}, \bar{x}_{j}\right) \geq 0
\end{aligned}
$$

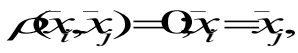

$$
\begin{aligned}
& \mu\left(x_{y}, \bar{x}_{j}\right)=\left(\bar{x}_{,}, \bar{x}_{y}\right) \text {, } \\
& \mu\left(x_{,}, \bar{x}_{j}\right) \leq \alpha\left(\bar{x}_{k}, \bar{x}_{k}\right)+\mu\left(x_{k}, \bar{x}_{j}\right)
\end{aligned}
$$

The developed approach a function of the distance is

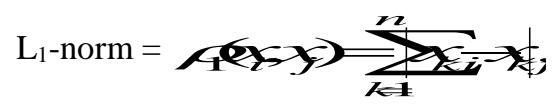

Options are analyzed for similarity measure defined as a fuzzy, non-negative real function

$$
S\left(\bar{x}_{i}, \bar{x}_{j}\right)=S_{i j}
$$

and satisfying the following conditions when comparing variants $\mathrm{i}$ and $\mathrm{j}$ : 


$$
\begin{aligned}
& 0 \leq S\left(\bar{x}_{i}, \bar{x}_{j}\right) \leq 1, \\
& S\left(\bar{x}_{i}, \bar{x}_{j}\right)=1, \\
& S\left(\bar{x}_{i}, \bar{x}_{j}\right)=S\left(\bar{x}_{j}, \bar{x}_{i}\right), \\
& x_{i} \neq x_{j} .
\end{aligned}
$$

Similarity measure is based on the distance measures:

$$
\begin{aligned}
S_{i j} & =\frac{1}{1+\rho_{i j}^{2}}, \\
S_{i j} & =1 \rightarrow \rho_{i j}=0, \\
S_{i j} & =0 \rightarrow \rho_{i j}=\infty .
\end{aligned}
$$

Field of study further narrows to several clusters, which are then investigated. To increase the degree of descriptiveness when choosing a converged variants $X_{R k}$ are generated, i.e. variants with a maximum score for each of the criteria and "best" or "ideal" version of $\mathrm{X}_{\mathrm{RI}}$, i.e. ES having a maximum score. To compare the different options, the morphological space of solutions is constructed, in which the position of ES describes the properties of selected variants (figure 7). The sought-for ES $\mathrm{x} *$ is searched for among the many options $\mathrm{X}_{\mathrm{R}}$

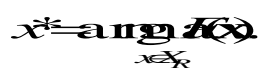

First reviewed the most promising options and clusters with the highest scores or similar in layout to the reference variants.

Comparing the variants of ES we determine the best ones of which the final table is made, where, after all samples are taken a set of acceptable ES is left. At the output structural schemes ("white boxes") ES are generated, consisting of models of composition and structure. According to the basic provisions of the methodology software complexes of structural optimization "Okkam" is developed. Below example of realization of advanced morphological approach.

Clusters

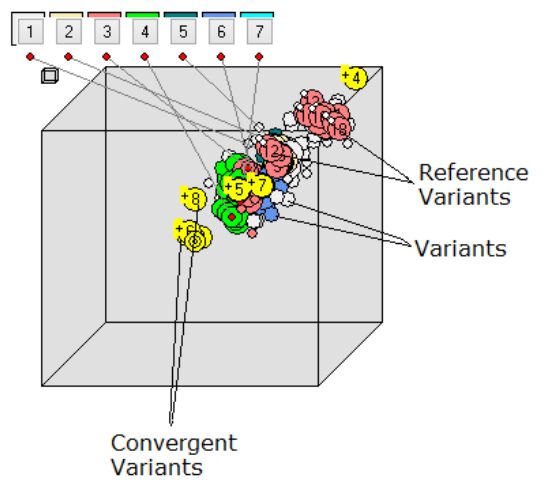

Fig. 7. Clustering of variants and the solution space 


\section{Advanced morphological approach to finishing automation of GTE blades}

In particular, the clusters of ESs were investigated. The matrix was constructed that contained about 560 thousand potential variants [12]. Some of the feasible engineering solutions that were generated using morphological approach are shown in figure 8 . Engineering system that realizes above mentioned technique of finishing operation for gas turbine engine blades is presented in figure 9.

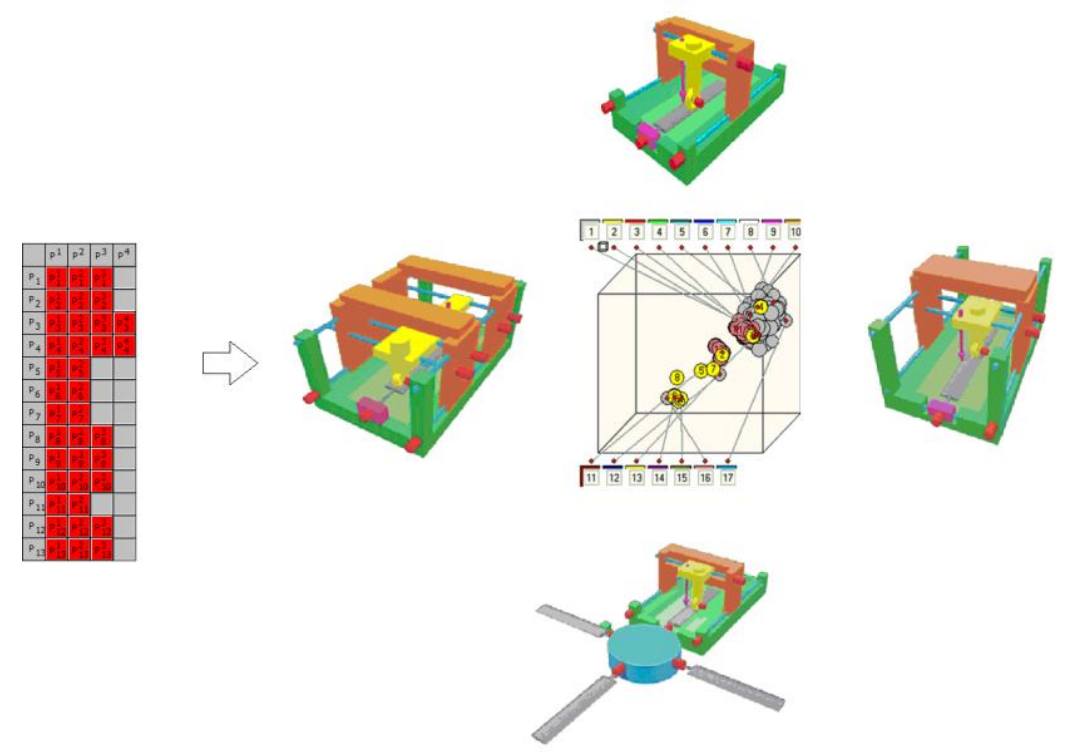

Fig. 8. MM and feasible engineering solutions of machine tool for GTE engine blades

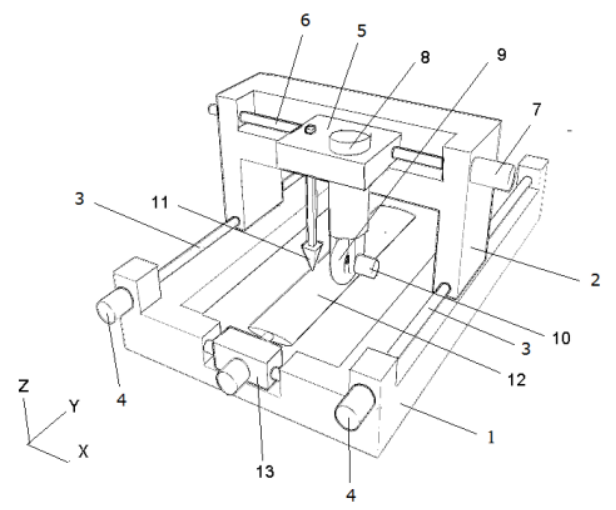

Fig. 9. Portal-type machine tool for finishing machining of GTE blades 


\section{Summary And Conclusion}

A new advanced morphological approach of structural analysis and synthesis of structural ES is proposed, which allows to improve the quality and efficiency of work in the creation of new systems. The approach allows to search for new engineering solutions during the conceptual design phase, to form clusters of options, to generate a set of pre-optimal options, to choose the most rational variants and to compare them. It is based on the positions of system, cluster analysis and morphological approach. As the approach has a generic character it is possible to apply it systematically to identify robust solutions for complex engineering challenges.

The AMA clarifies and organizes the structuring of the deciding task with the validity of decision making is increased while a multitude of variants, among which the selection is carried out, can be handled. This improves the process quality to select/identify the rational engineering systems to be developed. The software OKKAM can be implemented into CAI and use as part Knowledge Engineering and Data Mining.

\section{References}

1. V.P.Mishin, M. I. Osin, Introduction to Aircrafts Design, Mashinostroenie (1978)

2. D. Rakov, A Sinev, The structural analysis of new technical systems based on a morphological approach under uncertainty conditions, J. of Machinery Manufacture and Reliability 7, 74-81 (2015)

3. V. Hubka, W.Eder, Theory of technical systems: A total concept theory for engineering design, Springer Verlag (1988)

4. A. Polovinkin, Design theory of new engineering solutions: technology patterns and their applications, Edn. Informelectro (1991)

5. M. Levin, Modular System Design and Evaluation, Springer International Publishing (2015)

6. M. Smerlinski, M. Stephan, C. Gundlach, Innovationsmanagement in hessischen Unternehmen. Eine empirische Untersuchung zur Praxis in klein- und mittelständischen Unternehmen, Marburg (2009)

7. F. Zwicky, Discovery, Invention, Research - Through the Morphological Approach, Toronto, The Macmillan Company (1969)

8. T. Ritchey, Problem Structuring using computer-aided morphological analysis, J. of the Operational Research Society, Special Issue on Problem Structuring Methods, 57 792-801 (2006)

9. B. Garvey, Combining quantitative and qualitative aspects of problem structuring in computational morphological analysis, $\mathrm{PhD}$ thesis, Imperial College London (2016)

10. B. Klimenko, D Rakov, Analysis and Synthesis of Innovative Engineering Solutions and Technologies Based on Advanced Morphological Approach, Advances in Intelligent Systems and Computing, 658, 274-283 (2018)

11. A. Bardenhagen, D.Rakov, Advanced morphological approach in Aerospace design during conceptual stage. Online First article, accepted for publication in Facta Universitas (2019)

12. I. Kondrat'ev, D. Rakov, Advanced morphological approach to finding novel solutions for automated finishing of GTE blades, Mechanical Science and Technology Update, IOP Conf. Series: Journal of Physics: Conf. Series, 1260, (2019) 